\title{
Longitudinal bidirectional relationships between maternal depressive/anxious symptoms and children's tics
}

Tomoko Yagi ( $\square$ tomokoyagi1112@gmail.com )

The University of Tokyo

Shuntaro Ando

The University of Tokyo

Satoshi Usami

The University of Tokyo

Syudo Yamasaki

Tokyo Metropolitan Institute of Medical Science

Masaya Morita

The University of Tokyo

Tomoki Kiyono

The University of Tokyo

Noriyuki Hayashi

The Univeristy of Tokyo

Kaori Endo

Tokyo Metropolitan Institute of Medical Science

Yudai lijima

Tokyo Metropolitan Institute of Medical Science

Yuko Morimoto

The Graduate University of Advanced Studies

Sho Kanata

Teikyo University School of Medicine

Shinya Fujikawa

The University of Tokyo

Shinsuke Koike

The University of Tokyo

Yukiko Kano

The University of Tokyo

Mariko Hiraiwa-Hasegawa

The Graduate University of Advanced Studies

Atsushi Nishida

Tokyo Metropolitan Institute of Medical Science

Kiyoto Kasai

The University of Tokyo

Research article

Keywords: Tics, Maternal depressive/anxious symptoms, Longitudinal study, General population study, Early adolescence

Posted Date: January 15th, 2021

DOI: https://doi.org/10.21203/rs.3.rs-17370/v2

License: (i) This work is licensed under a Creative Commons Attribution 4.0 International License. Read Full License 


\section{Abstract}

Background: Previous studies have revealed an association between maternal depressive/anxious symptoms and children's tics. However, the longitudinal relationships between these symptoms remain unclear. We examined the longitudinal relationships between maternal depressive/anxious symptoms and children's tics across early adolescence in a population-based sample.

Methods: The participants consisted of 3,171 children and their mothers from the Tokyo Teen Cohort (TTC) study, a population-representative longitudinal study that was launched in Tokyo in 2012. Maternal depressive/anxious symptoms and children's tics were examined using self-report questionnaires at the ages of 10 (time 1, T1) and 12 (time 2, T2). A cross-lagged model was used to explore the relationships between maternal depressive/anxious symptoms and children's tics.

Results: Higher levels of maternal depressive/anxious symptoms at T1 were related to an increased likelihood of children's tics at T2 ( $\beta=.06, p=.001)$. Furthermore, the presence of children's tics at T1 was positively related to maternal depressive/anxious symptoms at T2 ( $\beta=.06, p=.001)$.

Conclusions: These findings suggest a longitudinal bidirectional relationship between maternal depressive/anxious symptoms and children's tics during early adolescence that may exacerbate each other over time and possibly create a vicious cycle. When an early adolescent has tics, it might be important to identify and treat related maternal depressive/anxious symptoms.

\section{Background}

Tics are sudden, rapid, recurrent, and nonrhythmic motor movements or vocalizations. The Diagnostic and Statistical Manual of Mental Disorders, 5th edition (DSM-5) includes three tic disorders (1). Tourette syndrome (TS) is defined by the presence of at least two motor tic behaviors and one vocal tic behavior for a minimum period of a year, manifesting before the age of 18. Chronic tic disorder (CT) is defined by the presence of either motor or vocal tics for at least 1 year, while provisional tic disorder is defined as tics that have been present for less than a year. Recent population-based studies have demonstrated that tics are more common than previously recognized (2-5). According to the International Classification of Diseases 10th Revision (ICD-10), which is an international diagnostic classification developed by the World Health Organization (WHO), one in five to ten children has experienced tics (6). Tic disorders impose a psychosocial burden on children and their families because tics are characterized by the visibility of symptoms, which can cause stigma and prejudice (7-10). Attention deficit hyperactivity disorder (ADHD) and obsessive compulsive disorder (OCD) are common comorbidities of tic disorders (7, 11, 12). Tic disorders tend to be remitted with age through adolescence $(7,13,14)$. The overall similarity in these patterns of comorbidity and natural history among tic disorders suggests that tic disorders have etiological continuity (15-17), and a recent diagnosis of "tic spectrum disorders" has been suggested (18).

Tic disorders consist of a complex involvement of both multiple genes and environmental factors $(19,20)$; therefore, the identification of parental psychopathology could be informative in the evaluation of risk factors for the development of tic disorders in children (21). Previous research has shown that there is an association between maternal psychiatric symptoms and children's tic disorders. Postnatal maternal anxious symptoms and prenatal maternal depressive symptoms have been associated with increased odds of children having TS/CT at age 13 (22). In another study, a maternal history of nonspecific psychiatric disorders, including anxiety disorders and depressive disorders, was shown to increase odds of children having TS/CT during childhood and adolescence (23). It is presumed that maternal depressive/anxious symptoms are associated with the occurrence of children's TS/CT via maternal-specific environmental and/or genetic factors $(22,23)$.

Although the association between maternal mental health and children's tic disorders has been clarified, it is not clear whether maternal psychiatric symptoms are associated with the subsequent course of children's tics. If maternal psychiatric symptoms predict the subsequent course of children's tics, then maternal psychiatric symptoms could possibly be a prognostic factor or an intervention target of tics. To examine this point, we investigated the relationship between maternal depressive/anxious symptoms and children's tics with a longitudinal design.

In addition, we speculated that maternal depressive/anxious symptoms and children's tics influence each other bidirectionally. Some studies have shown bidirectional influences on maternal and children's psychiatric symptoms, whereby depression in mothers increases the risk of emotional and behavioral problems in their children and vice versa $(24,25)$. However, no study has investigated the bidirectional relationship between maternal psychiatric symptoms and children's tic disorders. Research on the longitudinal bidirectional relationship between maternal depressive/anxious symptoms and children's tics would be helpful in advancing the research of and practices related to tics.

Our aim was therefore to examine the relationships between maternal depressive/anxious symptoms and children's tics in a longitudinal study design using a general population of early adolescent samples. In this population-based study, we referred to "tics" instead of the diagnostic term "tic disorders" because we did not make clinical diagnoses of the participants. Our hypotheses were as follows: 1) maternal depressive/anxious symptoms predict the presence of children's tics two years later, and 2) maternal depressive/anxious symptoms and children's tics influence each other bidirectionally over time.

\section{Method}

\section{Participants}

This study used data from the Tokyo Teen Cohort (TTC) study (http://ttcp.umin.jp/), a population-based longitudinal survey focusing on children's health from biopsychosocial multidisciplinary viewpoints (26). The TTC study has started from October 2012 and is currently being conducted. The participants were recruited from three municipalities in Tokyo (Setagaya, Mitaka, and Chofu) using the Basic Resident Register. The candidate participants were 14,553 children born between September 1, 2002, and August 31, 2004. Invitation letters were sent to the primary parents of those children around their tenth birthday. Of 
these children, 10,234 were successfully contacted, and these children were invited to participate in the cohort study. Of these 10,234 children, 4,478 children participated in the baseline survey named the Tokyo Early Adolescence Survey (T-EAS). This baseline survey was conducted from October 2012 to January

2015, when the participants were approximately 10 years old (time 1, T1). Among the 4,478 participants in the T-EAS, candidates were chosen as participants for the second wave of the TTC study. For the sake of cohort management, the target number of participants to be included in the second wave of the TTC was 3,000 children. When choosing these TTC participants, an oversampling method was used instead of inclusion criteria, considering the low follow-up rate of families with low annual household incomes. Thus, 3,171 participants were extracted as targets for the second wave of the TTC study. This second wave of the TTC study was carried out from August 2014 to December 2016, at the time when the participants were approximately 12 years old (time 2, T2). Of the 3,171 children who were invited, 3,007 individuals participated in the second wave of the TTC study (follow-up rate $94.8 \%$ ). In each wave of the data collection, trained interviewers visited the participants' homes. They distributed questionnaires to the children and primary parents (mostly mothers), and they conducted psychological tests on the children.

\section{Ethical approval}

Ethical approval for this study was obtained from the research ethics committees of the Tokyo Metropolitan Institute of Medical Science (Approval number: 12-35), The Graduate University for Advanced Studies, SOKENDAI (2012002), and the Graduate School of Medicine and Faculty of Medicine, The University of Tokyo (10057). We obtained informed assent from the children and written informed consent from their primary parents.

\section{Measures}

\section{Tics}

We evaluated tics at T1 and T2. The participants' primary parents answered a questionnaire about the children's tics; this questionnaire has been used in a previous study (5). The questionnaire includes a section with the following five questions about specific motor and vocal tics in the past year: "Q1: Has your child had any repeated movements of parts of the face and head (e.g., eye blinking, grimacing, sticking tongue out, licking lips, spitting)?”; "Q2: Has your child had repeated movements of the neck, shoulder or trunk (e.g., twisting around, shoulder shrugging, bending over, nodding)?"; “Q3: Has your child had repeated movements of the arms, hands, legs, or feet?"; "Q4: Has your child had repeated noises and sounds (e.g., coughing, clearing throat, grunting, gurgling, hissing)?"; and "Q5: "Has your child had repeated words or phrases?" Each question is answered as either "definitely", "probably" or "not at all" present. Furthermore, we asked the following question about the frequency of these repetitive behaviors: "Q6: About how often does/did this happen in the last year?" This question was answered on the following 5-point Likert scale: "1: less than once a month, 2: 1-3 times a month, 3: about once a week, 4: more than once a week, 5: every day."

We evaluated the presence of tics using binary variables consisting of either "with tics" or "without tics." We defined the participants who responded "definitely" or "probably" to any of Q1, Q2, and Q4 as having tics. The participants who only endorsed repeated movements of the arms, hands, legs or feet (Q3) or repeated words or phrases (Q5) in the absence of a positive response to the other questions about the types of tics (Q1, Q2, Q4) were excluded from all case definitions to remove nontic movements such as stereotypy or isolated echolalia. We defined as tics all responses of "definitely" or "probably" to questions concerning motor and/or vocal tics regardless of their frequency because there is no condition of frequency in the diagnostic criteria of tic disorders (1) and because we aimed to exhaustively find tics in the general population.

In the post hoc analysis, we used a narrower definition of tics that required answers of "definitely" or "probably" to questions concerning motor and/or vocal tics and frequencies of "daily" or "more than once a week." This narrower definition of tics referred to the "broad definition of TS and CT" used in a previous population-based study investigating the prevalence of TS/CT (5). The "broad definition of TS and CT" used in this previous study differed from the narrower definition of tics used in this study in that the previous study was carried out on participants aged 13 and excluded subjects with an intellectual disability or autism.

\section{Maternal depressive/anxious symptoms}

We employed the Kessler Psychological Distress Scale (K6) (27-29) for T1 and the General Health Questionnaire-28 (GHQ-28) (30, 31) for T2. The K6 and the GHQ-28 are both widely used self-report questionnaires that were developed to evaluate depressive/anxious symptoms. We used different scales between T1 and T2 in the current study because the TTC study also switched the scale used for maternal depressive/anxious symptoms from the K6 to the GHQ-28 starting at T2. The $\mathrm{K} 6$ is a short questionnaire consisting of 6 questions about the subjective mental distress of the respondent over the past 30 days that are answered on a 5-point scale, and the scores of the 6 items are added together (0-24 points). The GHQ-28 consists of 28 questions about the respondent's subjective physical and mental states over the past few weeks, with a total score being calculated for each item by giving 0 points each for the right two responses and 1 point each for the left two responses (0-28 points). Cutoff values are often used to screen for anxiety disorders and depression when assessing the K6 and the GHQ-28. However, in this study, we used raw values of the K6 and the GHQ-28 as continuous scales instead of screening scales, for the purpose of evaluating the severity of depressive/anxious symptoms, including the normal range in the general population. The Cronbach's alpha value

Page 3/13 
was .84 for the $\mathrm{K} 6$ and .88 for the GHQ-28. We found that the distributions of the K6 and the GHQ-28 were similar based on the graphing cumulative distribution of their $\mathrm{Z}$ scores (Figure 1). If a primary parent other than a mother answered the K6 or the GHQ-28, we regarded those responses as missing values.

\section{Other variables}

Sex $(5,7,32)$, age (33-35), maternal age (23, 36-38), socioeconomic status (39), and maternal alcohol use during pregnancy (40) were included in the analyses since previous studies have reported that these factors influence the occurrence of TS/CT. The data for these variables were obtained from the responses to the questionnaires completed by caregivers. To assess socioeconomic status, family income was evaluated on a 10-point scale, which ranged from "0990,000 yen" to "more than 10,000,000 yen." Information on maternal alcohol use during pregnancy was obtained from maternity record books that were provided for almost all mothers by local public organizations in Japan.

\section{Statistical analysis}

Longitudinal relationships between maternal depressive/anxious symptoms and children's tics were studied with structural equation modeling. We used SPSS ${ }^{\circledR}$ (Statistical Package for Social Science; IBM Corp., Armonk, N.Y. USA) version 21.0 for the characteristics of the study participants and Amos ver. 22.0 (IBM Corp, New York) for the structural equation modeling. We used the following three cross-lagged design models. The first model analyzed the longitudinal relationships between maternal depressive/anxious symptoms and children's tics without adjusting for covariates (unadjusted model). The second model adjusted for sex, age in months, family income, maternal age, and maternal alcohol use during pregnancy (adjusted model). Finally, the third model took into account the nonnormal distribution of particular dependent variables (maternal depressive/anxious symptoms and children's tics at T2), logarithmically converted these measures and then conducted an analysis (logarithmically transformed model).

We also conducted a post hoc analysis by using the narrower definition of tics, which referred to the "broad definition of TS and CT" used in a previous study about the prevalence of TS/CT (5). The aims of the post hoc analysis were to compare the prevalence of tics in this study with that from the previous study and to examine the stability of the results on the relationships between maternal depressive/anxious symptoms and children's tics.

Missing values in the categories of tics, maternal depressive/anxious symptoms, and the covariates were accounted for by full information maximum likelihood procedures available in Amos. This method estimates model parameters and standard errors using all available data while adjusting for the uncertainty associated with missing data (41).

The threshold for statistical significance was set to $p<.05$ (two-sided) for all analyses. We evaluated the fit of our models by using the comparative fit index (CFI) and the root mean square error of approximation (RMSEA). A good model fit was indicated by an RMSEA value smaller than .05 and a CFI value larger than $.95(42,43)$.

\section{Results}

\section{Characteristics of the study participants}

Table 1 shows the demographic characteristics of the 3,171 study participants. Of the 3,171 included children, 2,601 children (82.0\%) had complete data about tics across both time points; 67 (2.1\%) and 484 (15.3\%) children had missing data about tics in either T1 or T2, respectively, and 19 ( $0.6 \%)$ children had missing data about tics in both $\mathrm{T} 1$ and $\mathrm{T} 2$. Across both time points, data about maternal depressive/anxious symptoms were complete for 2,683 mothers (84.6\%); 24 (0.8\%) and 309 (9.7\%) mothers had missing scores in either T1 or T2, respectively; and 155 mothers (4.9\%) had missing data about maternal depressive/anxious symptoms in both $\mathrm{T} 1$ and $\mathrm{T} 2$.

Of the participants, $23.9 \%$ children (744 of 3,109 available data) at T1 and $23.6 \%$ children (632 of 2,674 available data) at T2 had tics. These prevalence rates are consistent with previous studies that have estimated the point prevalence of tics in childhood to be approximately $20-29 \%$ (2-4). Comparing the presence of tics between T1 and T2 using McNemar's test, we found no significant difference $(p=.42)$. Of the 2,612 people whose data on the presence of tics were obtained in both T1 and T2, 343 participants endorsed tics at both T1 and T2, 290 participants endorsed tics only for T1, 270 participants endorsed tics for only $\mathrm{T} 2$, and 1,724 did not endorse tics at either time point.

\section{Longitudinal relationships between maternal depressive/anxious symptoms and children's tics}

We investigated the relationships between maternal depressive/anxious symptoms and children's tics in a cross-lagged model analysis (Figure 2, Table 2). There was a cross-sectional association between maternal depressive/anxious symptoms and children's tics at T1 and T2. Higher levels of maternal depressive/anxious symptoms at T1 significantly increased the presence of tics at T2 (adjusted model: $\beta=.06, p=.001$ ). In contrast, the presence of tics at 
T1 was related to higher levels of maternal depressive/anxious symptoms at T2 (adjusted model: $\beta=.06, p=.001$ ). All of these models indicated acceptable model fit to the data (adjusted model: $\mathrm{CFI}=.951$, RMSEA $=.046$ ). These results revealed that maternal depressive/anxious symptoms and children's tics had longitudinal, bidirectional relationships with each other.

\section{Post hoc analysis: analysis with a narrower definition of tics}

We also analyzed the cross-lagged design models with the narrower definition of tics which required the frequency of tics to be either "daily" or "more than once a week". This narrower definition referred to the "broad definition of TS and CT" used in a previous population-based study that investigated the prevalence of TS/CT (5). In this previous study, the prevalence rate of the "broad definition of TS and CT" was reported to be $11.8 \%$ at the age of 13 (5). Using the narrower definition of tics, $14.0 \%$ of the participants at the age of 10 (431 out of 3,085 effective data) and $12.1 \%$ of the participants at the age of 12 ( 323 out of 2,668 effective data) had tics among participants in this study. The presence of tics at T2 was significantly less than that in T1 ( $p=.003)$. Furthermore, we found longitudinal, bidirectional relationships between maternal depressive/anxious symptoms and children's tics (Table 3).

\section{Discussion}

This was the first study to examine the longitudinal relationships between maternal depressive/anxious symptoms and children's tics in a population-based early adolescent sample. The following two findings were obtained. First, when more severe maternal depressive/anxious symptoms are present, children are more likely to present tics two years later. Second, the severity of maternal depressive/anxious symptoms and the presence of children's tics are longitudinally associated with each other.

Several explanations may be possible for the significant magnitude-response relationship between maternal depressive/anxious symptoms and children's later tics. First, maternal depressive/anxious symptoms may affect the occurrence, persistence, and exacerbation of children's tics as an environmental factor because environmental factors such as psychosocial stresses are known to exacerbate tics (44-48). There are no reports of an association between maternal psychiatric symptoms and the course of children's tics. However, some studies have shown an association between maternal psychiatric symptoms and the course of children's psychiatric symptoms. For example, maternal depression has been shown to be associated with increased psychiatric diagnoses and emotional and behavioral problems in children, and when maternal depression is remitted, the children's problems are more likely to also be in remission (49). Furthermore, improvement in parental depression has a positive impact on the health, emotional, cognitive, academic and overall functioning of children (50). Thus, similar to these reports, maternal depressive/anxious symptoms might affect the course of children's tics as an environmental factor. Second, there might be genetic relationships between maternal depressive/anxious symptoms and the occurrence, persistence, and exacerbation of tics. Family studies of TS have suggested that TS has genetic correlations with depressive disorders and anxiety disorders $(51,52)$, although these correlations are possibly mediated through ADHD and OCD (52). There has been no research examining the genetic relationships between maternal depressive/anxious symptoms and the persistence or exacerbation of tics, but the results of this research could not rule out these possibilities. Furthermore, there is also a possibility that an interaction of genetic and environmental factors is involved in the relationships between maternal depressive/anxious symptoms and children's tics. The results of this study could not distinguish genetic and environmental contributors to the relationships between maternal depressive/anxious symptoms and children's tics.

Our findings on the association between the presence of tics and increased maternal depressive/anxious symptoms two years later can be explained by several potential mechanisms. One of the possible mechanisms is that the parenting stress associated with bringing up children with tics might influence maternal depressive/anxious symptoms. Parents of children with TS experience increased levels of caregiver burdens and parenting stress compared to parents of children without TS $(53,54)$. The visible nature of tics can have an impact on the parent-child relationship, with parents becoming overprotective of, worrying about, struggling to accept or trying to control children's tics, which can lead to family conflicts, poor parent-child relationships and increased frustrations in parenting $(7,9,10,55,56)$. Parenting stress in parents of children with tics could also occur due to children's comorbidities, such as $\mathrm{ADHD}, \mathrm{OCD}$, and behavioral problems $(53,54)$. In a previous population-based study, $21.2 \%$ of children had tics, and children with tics were more affected by psychopathologies, including ADHD and OCD, than were children without tics (12). In addition to these environmental factors, both genetic factors and genetic/environmental interactions might have an effect on children's tics on maternal depressive/anxious symptoms.

In a post hoc analysis, the prevalence rates of tics found by using the narrower definition of tics were $14.0 \%$ at the age of 10 and $12.1 \%$ at the age of 12. This narrower definition of tics referred to the "broad definition of TS and CT" used in a previous study, and the prevalence rate of "broad definition of TS and $\mathrm{CT}^{\prime \prime}$ has been reported to be $11.8 \%$ at the age of 13 (5). The prevalence rates of tics in this study were concordant with those found in the previous study, considering that the previous study excluded subjects with an intellectual disability or autism (5) and that the prevalence of tics decreases slowly from early adolescence $(7,13,33,57-60)$. Furthermore, when using the narrower definition of tics, we also found longitudinal bidirectional relationships between maternal depressive/anxious symptoms and children's tics. Thus, we confirmed the stability of the results of the main analysis.

The implications of this study were that the longitudinal bidirectional relationships between maternal depressive/anxious symptoms and tics in early adolescence may suggest a vicious cycle in which maternal depressive/anxious symptoms make tics worse, and tics make maternal depressive/anxious symptoms worse. This study also suggested that not only intervention in children's tics but also intervention in maternal depressive/anxious symptoms might be important for the treatment of tics. However, the present study was unable to separate genetic and environmental factors in the association between tics and maternal depressive/anxious symptoms; therefore, further research is needed to determine the effect of intervention on maternal anxiety/depressive symptoms. While there has been a consensus on the importance of family psychoeducation in the treatment of tics $(7,60)$, it is not known whether maternal psychiatric problems influence the course of children's tics. This study provides new insights for future research and practice. 
The strength of this study was that, for the first time, it was shown that higher levels of maternal depressive/anxious symptoms are related to an increased likelihood of tics two years later and that there are longitudinal relationships between maternal depressive/anxious symptoms and children's tics. Other strengths were the large sample size, the high follow-up rate of the study, and the inclusion of nonclinical tics. In contrast, this study also had several limitations. First, we used different measures of maternal depressive/anxious symptoms for T1 and T2. We found that the distributions of the K6 and the GHQ-28 were similar by graphing the cumulative distribution of the Z scores of the K6 and the GHQ-28 (Figure 1). Additionally, this limitation did not influence the course from maternal depressive/anxious symptoms to children's tics. Second, in this study, maternal depressive/anxious symptoms and children's tics were evaluated not by direct clinical assessments but by questionnaires to caregivers. However, we deliberately chose rigorous tic definitions and sought to exclude participants with nontic movement disorders (e.g., stereotypies associated with autism or an intellectual disability, repetitive arm/leg movements that could be better explained by tremor or motor restlessness) (5). In this study, the prevalence of tics was $23.9 \%$ at age 10 and $23.6 \%$ at age 12 . These prevalence rates could be considered reasonable based on the following evidence. Point prevalence depends strongly on age; the highest rate is estimated to be approximately $20 \%$ at age $5-10$, and the lifetime prevalence is much higher (3). In previous studies that have directly observed children, tics were found in $29.2 \%$ of fourth-grade children in an elementary school in Washington D.C. (2) and in $21.2 \%$ of children aged $9-17$ years old (mean 13.1 years old) in Monroe County, Rochester, New York (4). The prevalence rates of tics in the present study were consistent with those found in these previous studies. Third, the data analysis in this study could not adjust for ADHD and OCD, which are frequently comorbid with tics. That may be because of the strong association of tics with ADHD and OCD. Future studies are needed to examine the effects of ADHD and OCD on the bidirectional relationships between maternal depressive/anxious symptoms and children's tics. The fourth limitation was that the research interval in this longitudinal study was relatively short. Typically, tics improve gradually during adolescence, with repeated periods of remission and exacerbation. Thus, it might be difficult to capture change in the short research period of two years. Longer-term follow-up periods are needed in the future. The fifth limitation was that we did not collect information about the maternal history of tics. Given the low rate of medical consultation for tics $(5,12,51)$ and the clinical outcome that tics often improve or disappear after adolescence $(7,13,14)$, it is probably not possible to obtain accurate information on the maternal history of tics. Finally, there were also some limitations inherent to the cross-lagged model (61); i.e., there is a possibility that there are multiple potential additional factors (not included in the model) that influence the bidirectional relationship over time.

The following two studies would be helpful in testing the viability of the relationships between maternal depressive/anxious symptoms and children's tics and in advancing research and practice. First, if the course of children's tics could be observed at three or more time points, it would be possible to confirm the vicious cycle that develops between maternal depressive/anxious symptoms and children's tics and to investigate the mediating factors. For example, children's anxiety/depression symptoms might mediate the relationships between maternal depressive/anxious symptoms and children's tics. In addition, poor quality of life or comorbidities might mediate the relationships between children's tics and maternal depressive/anxious symptoms. Second, intervention studies could examine whether improvements in maternal depressive/anxious symptoms improve children's tics.

This study was the first to show the relationship between preceding maternal depressive/anxious symptoms and an increased risk of children's tics two years later in early adolescence. Furthermore, we found longitudinal bidirectional relationships between maternal depressive/anxious symptoms and children's tics. Although we could not separate environmental factors and genetic factors in this research, the findings implied that it may be important to care not only for children with tics but also for their mothers' depressive/anxious symptoms when tics are treated in early adolescence.

\section{Conclusion}

Previous studies have revealed the association between maternal depressive/anxious symptoms and children's tics. However, few studies have examined this association longitudinally; thus, the effect of maternal depressive/anxious symptoms on the course of tics has remained unknown. We examined the longitudinal relationships between maternal depressive/anxious symptoms and children's tics across early adolescence in a population-based sample. Our results demonstrated that with more severe maternal depressive/anxious symptoms, children are more likely to present tics two years later. This study also implied a longitudinal bidirectional relationship between maternal depressive/anxious symptoms and children's tics during early adolescence, which may exacerbate each other over time and possibly create a vicious cycle. Although we could not separate environmental factors and genetic factors in this research, we can speculate that it may be important to care not only for children with tics but also for their mothers' depressive/anxious symptoms when tics are treated in early adolescence.

\section{Abbreviations}

ADHD: attention deficit hyperactivity disorder

CFI: comparative fit index

Cl: confidence interval

CT: chronic tic disorder

DSM-5: Diagnostic and Statistical Manual of Mental Disorders 5th edition

GHQ-28: General Health Questionnaire-28

K6: Kessler Psychological Distress Scale

OCD: obsessive compulsive disorder 
RMSEA: root mean square error of approximation

SD: standard deviation

TS: Tourette syndrome

TTC: Tokyo Teen Cohort

\section{Declarations}

\section{Ethics approval and consent to participate}

Ethical approval for this study was obtained from the research ethics committees of Tokyo Metropolitan Institute of Medical Science (Approval number: 12 35), SOKENDAI (The Graduate University for Advanced Studies) (2012002), and the Graduate School of Medicine and Faculty of Medicine, The University of Tokyo (10057). We obtained informed assent from children and written informed consent from primary parents prior to engaging them in research.

\section{Consent for publication}

Not applicable.

\section{Availability of data and materials}

The datasets used and/or analyzed during the current study are available from the corresponding author on reasonable request.

\section{Competing interests}

The authors declare that they have no competing interests.

\section{Funding}

This work was supported by Grant-in-Aid for Scientific Research on Innovative Areas (23118002 and 16H01689; Adolescent Mind \& Self-Regulation) from the Ministry of Education, Culture, Sports, Science and Technology of Japan. This study was also supported in part by JSPS KAKENHI Grant numbers JP16H06395, 16H06398, 16H06399, 16K15566, 16K21720 \& 17H05931, by UTokyo Center for Integrative Science of Human Behavior (CiSHuB), by the International Research Center for Neurointelligence (WPI-IRCN) at the University of Tokyo Institutes for Advanced Study (UTIAS), and by AMED under Grant Number JP17ek0109262. The funding source had no role in the preparation, review, or approval of the manuscript; and the decision to submit the manuscript for publication.

\section{Authors' contributions}

TY conceptualized and designed the study, drafted the initial manuscript, and reviewed and revised the manuscript, being supervised by SA, AN, SY, and YK designed the study and reviewed and revised the manuscript. KK critically reviewed the manuscript for important intellectual content. SU supervised the statistical analysis. All authors contributed to and have approved the final manuscript.

\section{Acknowledgements}

Not applicable.

\section{References}

1. American Psychiatric Association. The diagnostic and statistical manual of mental disorders. 5th ed. Washington D.C: American Psychiatric Publishing; 2013.

2. Snider LA, Seligman LD, Ketchen BR, Levitt SJ, Bates LR, Garvey MA, et al. Tics and problem behaviors in schoolchildren: prevalence, characterization, and associations. Pediatrics. 2002;110(2 Pt 1):331-6.

3. Black KJ, Black ER, Greene DJ, Schlaggar BL. Provisional Tic Disorder: What to tell parents when their child first starts ticcing. F1000Res. $2016 ; 5: 696$.

4. Kurlan R, McDermott MP, Deeley C, Como PG, Brower C, Eapen S, et al. Prevalence of tics in schoolchildren and association with placement in special education. Neurology. 2001;57(8):1383-8.

5. Scharf JM, Miller LL, Mathews CA, Ben-Shlomo Y. Prevalence of Tourette syndrome and chronic tics in the population-based Avon longitudinal study of parents and children cohort. J Am Acad Child Adolesc Psychiatry. 2012;51(2):192-201 e5. 
6. World Health Organization. Tenth revision of the international classification of diseases and related health problems. Geneva: World Health Organization; 1992.

7. Murphy TK, Lewin AB, Storch EA, Stock S. Practice parameter for the assessment and treatment of children and adolescents with tic disorders. J Am Acad Child Adolesc Psychiatry. 2013;52(12):1341-59.

8. Hoekstra PJ, Lundervold AJ, Lie SA, Gillberg C, Plessen KJ. Emotional development in children with tics: a longitudinal population-based study. Eur Child Adolesc Psychiatry. 2013;22(3):185-92.

9. Smith H, Fox JR, Trayner P. The lived experiences of individuals with Tourette syndrome or tic disorders: a meta-synthesis of qualitative studies. Br $\mathrm{J}$ Psychol. 2015;106(4):609-34.

10. Malli MA, Forrester-Jones R, Murphy G. Stigma in youth with Tourette's syndrome: a systematic review and synthesis. Eur Child Adolesc Psychiatry. 2016;25(2):127-39.

11. Leckman JF. Tourette's syndrome. Lancet. 2002;360(9345):1577-86.

12. Kurlan R, Como PG, Miller B, Palumbo D, Deeley C, Andresen EM, et al. The behavioral spectrum of tic disorders: a community-based study. Neurology. 2002;59(3):414-20.

13. Leckman JF, Bloch MH, King RA, Scahill L. Phenomenology of tics and natural history of tic disorders. Adv Neurol. 2006;99:1-16.

14. Bloch MH, Leckman JF. Clinical course of Tourette syndrome. J Psychosom Res. 2009;67(6):497-501.

15. Kurlan R, Behr J, Medved L, Como P. Transient tic disorder and the spectrum of Tourette's syndrome. Arch Neurol. 1988;45(11):1200-1.

16. Kurlan R, Eapen V, Stern J, McDermott MP, Robertson MM. Bilineal transmission in Tourette's syndrome families. Neurology. 1994;44(12):2336-42.

17. Peterson BS, Pine DS, Cohen P, Brook JS. Prospective, longitudinal study of tic, obsessive-compulsive, and attention-deficit/hyperactivity disorders in an epidemiological sample. J Am Acad Child Adolesc Psychiatry. 2001;40(6):685-95.

18. Muller-Vahl KR, Sambrani T, Jakubovski E. Tic disorders revisited: introduction of the term "tic spectrum disorders". Eur Child Adolesc Psychiatry. 2019;28(8):1129-35.

19. Cavanna AE, Servo S, Monaco F, Robertson MM. The behavioral spectrum of Gilles de la Tourette syndrome. J Neuropsychiatry Clin Neurosci. 2009;21(1):13-23.

20. Mataix-Cols D, Isomura K, Perez-Vigil A, Chang Z, Ruck C, Larsson KJ, et al. Familial Risks of Tourette Syndrome and Chronic Tic Disorders. A PopulationBased Cohort Study. JAMA psychiatry. 2015;72(8):787-93.

21. Coffey BJ. Persistent tics, Tourette syndrome, and psychopathology: where are we now, and where are we going? J Am Acad Child Adolesc Psychiatry. 2017;56(4):281-3.

22. Ben-Shlomo Y, Scharf JM, Miller LL, Mathews CA. Parental mood during pregnancy and post-natally is associated with offspring risk of Tourette syndrome or chronic tics: prospective data from the Avon Longitudinal Study of Parents and Children (ALSPAC). Eur Child Adolesc Psychiatry. 2016;25(4):373-81.

23. Leivonen S, Scharf JM, Mathews CA, Chudal R, Gyllenberg D, Sucksdorff D, et al. Parental psychopathology and Tourette syndrome/chronic tic disorder in offspring: a Nationwide case-control study. J Am Acad Child Adolesc Psychiatry. 2017;56(4):297-303 e4.

24. Elgar FJ, McGrath PJ, Waschbusch DA, Stewart SH, Curtis LJ. Mutual influences on maternal depression and child adjustment problems. Clin Psychol Rev. 2004;24(4):441-59.

25. Kuckertz JM, Mitchell C, Wiggins JL. Parenting mediates the impact of maternal depression on child internalizing symptoms. Depress Anxiety. 2018;35(1):89-97.

26. Ando S, Nishida A, Yamasaki S, Koike S, Morimoto Y, Hoshino A, et al. Cohort Profile: The Tokyo Teen Cohort study (TTC). Int J Epidemiol. 2019;48(5):1414-g.

27. Sakurai K, Nishi A, Kondo K, Yanagida K, Kawakami N. Screening performance of K6/K10 and other screening instruments for mood and anxiety disorders in Japan. Psychiatry Clin Neurosci. 2011;65(5):434-41.

28. Furukawa TA, Kawakami N, Saitoh M, Ono Y, Nakane Y, Nakamura Y, et al. The performance of the Japanese version of the K6 and K10 in the World Mental Health Survey Japan. Int J Methods Psychiatr Res. 2008;17(3):152-8.

29. Kessler RC, Berglund P, Demler O, Jin R, Merikangas KR, Walters EE. Lifetime prevalence and age-of-onset distributions of DSM-IV disorders in the National Comorbidity Survey Replication. Arch Gen Psychiatry. 2005;62(6):593-602.

30. Goldberg DP. The detection of psychiatric illness by questionnaire. A technique for the identification and assessment of non-psychotic psychiatric illness. London: Oxford University Press; 1972.

31. Goldberg DP, Hillier VF. A scaled version of the General Health Questionnaire. Psychol Med. 1979;9(1):139-45.

32. Stefanoff P, Wolanczyk T, Gawrys A, Swirszcz K, Stefanoff E, Kaminska A, et al. Prevalence of tic disorders among schoolchildren in Warsaw, Poland. Eur Child Adolesc Psychiatry. 2008;17(3):171-8.

33. Leckman JF, Zhang H, Vitale A, Lahnin F, Lynch K, Bondi C, et al. Course of tic severity in Tourette syndrome: the first two decades. Pediatrics. 1998;102(1 Pt 1):14-9.

34. Rutter M, Bishop D, Pine D, Scott S, Stevenson J, Taylor E. Rutter's child and adolescent psychiatry. 5th ed. London: Blackwell Press; 2010.

35. Leckman JF. Phenomenology of tics and natural history of tic disorders. Brain Dev. 2003;25 Suppl 1:S24-8.

36. Leivonen S, Voutilainen A, Chudal R, Suominen A, Gissler M, Sourander A. Obstetric and neonatal adversities, parity, and Tourette syndrome: a nationwide registry. J Pediatr. 2016;171:213-9. 
37. Leivonen S, Chudal R, Joelsson P, Ekblad M, Suominen A, Brown AS, et al. Prenatal maternal smoking and Tourette syndrome: a nationwide register study. Child Psychiatry Hum Dev. 2016;47(1):75-82.

38. McGrath JJ, Petersen L, Agerbo E, Mors O, Mortensen PB, Pedersen CB. A comprehensive assessment of parental age and psychiatric disorders. JAMA psychiatry. 2014;71(3):301-9.

39. Miller LL, Scharf JM, Mathews CA, Ben-Shlomo Y. Tourette syndrome and chronic tic disorder are associated with lower socio-economic status: findings from the Avon Longitudinal Study of Parents and Children cohort. Dev Med Child Neurol. 2014;56(2):157-63.

40. Mathews CA, Scharf JM, Miller LL, Macdonald-Wallis C, Lawlor DA, Ben-Shlomo Y. Association between pre- and perinatal exposures and Tourette syndrome or chronic tic disorder in the ALSPAC cohort. Br J Psychiatry. 2014;204(1):40-5.

41. Schafer JL, Graham JW. Missing data: our view of the state of the art. Psychol Methods. 2002;7(2):147-77.

42. Byrne BM. Structural equation modeling with AMOS: basic concepts, applications, and programming. 2nd ed. New York: Routledge; 2010.

43. Kline RB. Principles and practice of structural equation modeling. 2nd ed. New York: The Guilford; 2005.

44. Lin H, Katsovich L, Ghebremichael M, Findley DB, Grantz H, Lombroso PJ, et al. Psychosocial stress predicts future symptom severities in children and adolescents with Tourette syndrome and/or obsessive-compulsive disorder. J Child Psychol Psychiatry. 2007;48(2):157-66.

45. Hoekstra PJ, Steenhuis MP, Kallenberg CG, Minderaa RB. Association of small life events with self reports of tic severity in pediatric and adult tic disorder patients: a prospective longitudinal study. J Clin Psychiatry. 2004;65(3):426-31.

46. Chappell P, Riddle M, Anderson G, Scahill L, Hardin M, Walker D, et al. Enhanced stress responsivity of Tourette syndrome patients undergoing lumbar puncture. Biol Psychiatry. 1994;36(1):35-43.

47. Silva RR, Munoz DM, Barickman J, Friedhoff AJ. Environmental factors and related fluctuation of symptoms in children and adolescents with Tourette's disorder. J Child Psychol Psychiatry. 1995;36(2):305-12.

48. Surwillo WW, Shafii M, Barrett CL. Gilles de la Tourette syndrome: a 20-month study of the effects of stressful life events and haloperidol on symptom frequency. J Nerv Ment Dis. 1978;166(11):812-6.

49. Weissman MM, Pilowsky DJ, Wickramaratne PJ, Talati A, Wisniewski SR, Fava M, et al. Remissions in maternal depression and child psychopathology: a STAR*D-child report. JAMA. 2006;295(12):1389-98.

50. Gunlicks ML, Weissman MM. Change in child psychopathology with improvement in parental depression: a systematic review. J Am Acad Child Adolesc Psychiatry. 2008;47(4):379-89.

51. Khalifa N, von Knorring AL. Tourette syndrome and other tic disorders in a total population of children: clinical assessment and background. Acta Paediatr. 2005;94(11):1608-14.

52. Hirschtritt ME, Lee PC, Pauls DL, Dion Y, Grados MA, Illmann C, et al. Lifetime prevalence, age of risk, and genetic relationships of comorbid psychiatric disorders in Tourette syndrome. JAMA psychiatry. 2015;72(4):325-33.

53. Stewart SB, Greene DJ, Lessov-Schlaggar CN, Church JA, Schlaggar BL. Clinical correlates of parenting stress in children with Tourette syndrome and in typically developing children. J Pediatr. 2015;166(5):1297-302 e3.

54. Cooper C, Robertson MM, Livingston G. Psychological morbidity and caregiver burden in parents of children with Tourette's disorder and psychiatric comorbidity. J Am Acad Child Adolesc Psychiatry. 2003;42(11):1370-5.

55. Elstner K, Selai CE, Trimble MR, Robertson MM. Quality of Life (QOL) of patients with Gilles de la Tourette's syndrome. Acta Psychiatr Scand. 2001;103(1):52-9.

56. Eddy CM, Rizzo R, Gulisano M, Agodi A, Barchitta M, Cali P, et al. Quality of life in young people with Tourette syndrome: a controlled study. J Neurol. 2011;258(2):291-301.

57. Centers for Disease Control and Prevention. Understanding Tourette syndrome. 2018. https://www.cdc.gov/ncbddd/tourette/index.html. Accessed 12 Aug 2018.

58. Scahill L, Erenberg G, Berlin CM, Jr., Budman C, Coffey BJ, Jankovic J, et al. Contemporary assessment and pharmacotherapy of Tourette syndrome. NeuroRx. 2006;3(2):192-206.

59. Singer HS, Walkup JT. Tourette syndrome and other tic disorders. Diagnosis, pathophysiology, and treatment. Medicine. 1991;70(1):15-32.

60. Kurlan R. Clinical practice. Tourette's syndrome. N Engl J Med. 2010;363(24):2332-8.

61. Selig JP, Little TD. Autoregressive and cross-lagged panel analysis for longitudinal data. In: Laursen B, Little TD, Card NA, editors. Handbook of developmental research methods. New York: The Guilford Press; 2012. p. 265-278.

\section{Tables}

Table 1 Demographic characteristics of the participants 


\begin{tabular}{|c|c|c|c|c|c|}
\hline \multirow[t]{2}{*}{ Variables } & \multicolumn{2}{|c|}{$\begin{array}{c}\mathrm{T} 1 \\
\text { (10 years of age) }\end{array}$} & \multicolumn{2}{|c|}{$\begin{array}{c}\mathrm{T} 2 \\
(12 \text { years of age) }\end{array}$} & \multirow[t]{2}{*}{$p$ value $^{\mathrm{a})}$} \\
\hline & n/Mean & $(\%) / S D$ & $\mathrm{n} /$ Mean & $(\%) / S D$ & \\
\hline Sex, male & 1,684 & (53.1\%) & & & \\
\hline Age in months & 122.1 & 3.3 & 146.0 & 3.7 & \\
\hline Maternal age & 42.0 & 4.2 & & & \\
\hline \multicolumn{6}{|l|}{ Family income ${ }^{b)}$} \\
\hline$<5$ million yen & 620 & $(20.4 \%)$ & 448 & $(16.5 \%)$ & \\
\hline$\geq 5$ million yen, $<8$ million yen & 941 & $(31.0 \%)$ & 782 & $(28.8 \%)$ & \\
\hline$\geq 8$ million yen, $<10$ million yen & 568 & $(18.6 \%)$ & 518 & $(19.1 \%)$ & \\
\hline$\geq 10$ million yen & 917 & $(30.1 \%)$ & 970 & $(35.7 \%)$ & \\
\hline Maternal alcohol use during pregnancy & 748 & $(27.2 \%)$ & & & \\
\hline \multicolumn{6}{|l|}{ Presence of tics (in main analysis) ${ }^{\mathrm{C}}$ ) } \\
\hline With tics & 744 & $(23.9 \%)$ & 632 & $(23.6 \%)$ & .42 \\
\hline Without tics & 2,365 & $(76.1 \%)$ & 2,042 & $(76.4 \%)$ & \\
\hline \multicolumn{6}{|c|}{ Presence of tics (narrower definition in post hoc analysis) ${ }^{C}$ ) } \\
\hline With tics & 431 & $(14.0 \%)$ & 323 & $(12.1 \%)$ & $.003^{* *}$ \\
\hline Without tics & 2,654 & $(86.0 \%)$ & 2,345 & (87.9\%) & \\
\hline \multicolumn{6}{|l|}{ Maternal depressive/anxious symptoms } \\
\hline $\mathrm{T} 1, \mathrm{~K} 6$ & 2.9 & 3.3 & & & \\
\hline T2, GHQ-28 & & & 5.4 & 4.9 & \\
\hline
\end{tabular}

Abbreviations: SD, standard deviation.

cNemar's test was used to compare the presence of tics between T1 and T2.

imily income was evaluated on the 10-point scale described in the Method section and categorized into the four groups in this table.

e obtained total 3,109 data for the presence of tics at T1 in the main analysis and total 3,085 data for that in the post hoc analysis. This screpancy in the total numbers arose because, as described in the methods, the presence of tics in the main analysis did not require the datum out the frequency of tics while the presence of tics in the post hoc analysis required the datum about the frequency of tics.

${ }^{* *} p<.01$

Table 2 Relationships between maternal depressive/anxious symptoms and children's tics $(\mathrm{N}=3,171)$ 


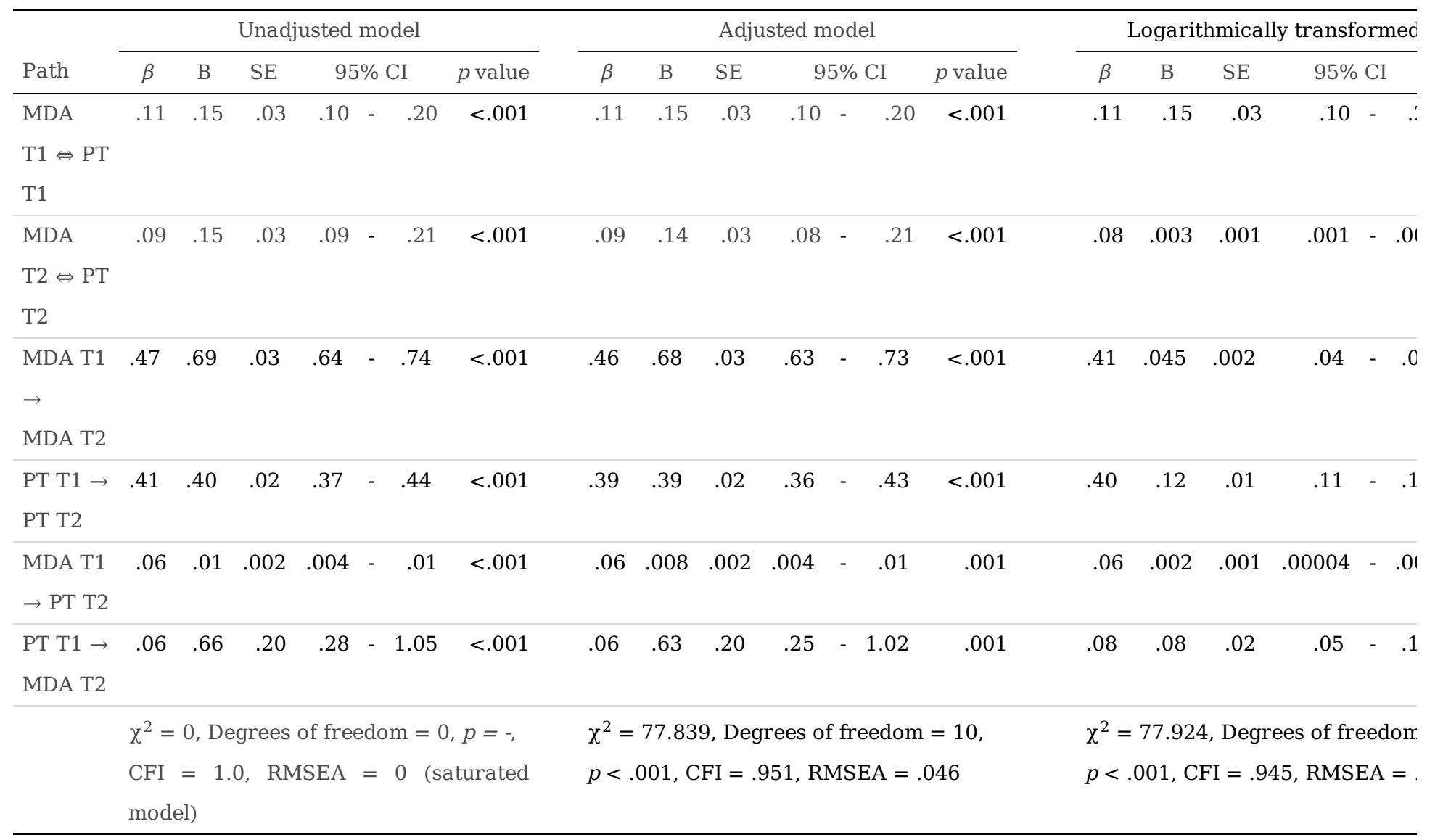

Abbreviations: MDA, maternal depressive/anxious symptoms; PT, presence of tics; $\beta$, standardized coefficient; B, nonstandardized coeffic standard error; 95\% CI, 95\% confidence interval; CFI, Confirmatory Fit Index; RMSEA, Root Mean Square Error of Approximation.

$\mathrm{T} 1,10$ years of age; T2, 12 years of age.

Table 3 Analysis with a narrower definition of tics: relationships between maternal depressive/anxious symptoms and children's tics ( $\mathrm{N}=$ 3,171) 


\begin{tabular}{|c|c|c|c|c|c|c|c|c|c|c|c|c|c|c|c|c|c|}
\hline \multirow{3}{*}{$\begin{array}{c}\text { Path } \\
\text { MDA }\end{array}$} & \multicolumn{6}{|c|}{ Unadjusted model } & \multicolumn{6}{|c|}{ Adjusted model } & \multicolumn{5}{|c|}{ logarithmically transforme } \\
\hline & \multirow{2}{*}{$\begin{array}{c}\beta \\
.08\end{array}$} & \multirow{2}{*}{$\begin{array}{c}\text { B } \\
.09\end{array}$} & \multirow{2}{*}{$\begin{array}{c}\text { SE } \\
.02\end{array}$} & \multicolumn{2}{|c|}{$95 \% \mathrm{CI}$} & \multirow{2}{*}{$\begin{array}{r}p \text { value } \\
<.001\end{array}$} & \multirow{2}{*}{$\begin{array}{c}\beta \\
.08\end{array}$} & \multirow{2}{*}{$\begin{array}{l}\text { B } \\
.09\end{array}$} & \multirow{2}{*}{$\begin{array}{c}\text { SE } \\
.02\end{array}$} & \multicolumn{2}{|c|}{$95 \% \mathrm{CI}$} & \multirow{2}{*}{$\begin{array}{r}p \text { value } \\
<.001\end{array}$} & \multirow{2}{*}{$\begin{array}{l}\beta \\
.08\end{array}$} & \multirow{2}{*}{$\begin{array}{l}\text { B } \\
.09\end{array}$} & \multirow{2}{*}{$\begin{array}{c}\text { SE } \\
.02\end{array}$} & \multicolumn{2}{|c|}{$95 \% \mathrm{CI}$} \\
\hline & & & & .05 & -.13 & & & & & .05 & -.13 & & & & & .05 & $-\quad .1$ \\
\hline \multicolumn{18}{|l|}{$\mathrm{T} 1 \Leftrightarrow \mathrm{PT}$} \\
\hline \multicolumn{18}{|l|}{$\mathrm{T} 1$} \\
\hline MDA & .07 & .09 & .03 & .04 & -.14 & $<.001$ & .07 & .09 & .03 & .04 & $-\quad .14$ & $<.001$ & .06 & .002 & .001 & .00004 & -.00 \\
\hline \multicolumn{18}{|l|}{$\mathrm{T} 2 \Leftrightarrow \mathrm{PT}$} \\
\hline \multicolumn{18}{|l|}{$\mathrm{T} 2$} \\
\hline MDA T1 & .47 & .69 & .03 & .64 & -.74 & $<.001$ & .46 & .68 & .03 & .64 & $\begin{array}{l}-\quad .73 \\
\end{array}$ & $<.001$ & .41 & .05 & .002 & .04 & -.0 \\
\hline \multicolumn{18}{|l|}{$\rightarrow$} \\
\hline \multicolumn{18}{|l|}{ MDA T2 } \\
\hline PT T1 & .34 & .32 & .02 & .29 & -.36 & $<.001$ & .34 & .32 & .02 & .28 & $-\quad .35$ & $<.001$ & .34 & .10 & .01 & .09 & -.11 \\
\hline \multicolumn{18}{|l|}{$\rightarrow$} \\
\hline \multicolumn{18}{|l|}{ PT T2 } \\
\hline MDA T1 & .05 & .01 & .002 & .001 & $\begin{array}{l}-\quad .01 \\
\end{array}$ & .005 & .05 & .005 & .002 & .001 & $\begin{array}{ll}- & .01\end{array}$ & .008 & .05 & .001 & .001 & .0003 & -.00 \\
\hline \multicolumn{18}{|l|}{$\rightarrow \mathrm{PT} \mathrm{T2}$} \\
\hline PT T1 & .06 & .85 & .24 & .37 & - 1.33 & $<.001$ & .06 & .82 & .24 & .34 & - 1.30 & $<.001$ & .07 & .07 & .02 & .04 & $-\quad .1$ : \\
\hline \multicolumn{18}{|l|}{$\rightarrow$} \\
\hline \multicolumn{18}{|l|}{ MDA T2 } \\
\hline & $\chi^{2}=$ & $0, \mathrm{De}$ & grees & of free & $\mathrm{dom}=\mathrm{c}$ & $p=-$ & $\chi^{2}=$ & 77.84 & 0, Deg & rees o & freedom & $=10$ & $\chi^{2}=$ & 77.93 & 5, Degl & ees of fre & eedor. \\
\hline & $\mathrm{CFI}=$ & $=1.0$, & RMSE & $A=0$ & (saturat & ed & $p<$. & 001, & & $\mathrm{CFI}=$ & $.943, \mathrm{RN}$ & SEA = & .001, & & & & \\
\hline & mode & l) & & & & & .046 & & & & & & $\mathrm{CFI}=$ & $=.934$ & RMSE & $A=.046$ & \\
\hline
\end{tabular}

Abbreviations: MDA, maternal depressive/anxious symptoms; PT, presence of tics; $\beta$, standardized coefficient; $\mathrm{B}$, nonstandardized coeffic standard error; 95\% CI, 95\% confidence interval; CFI, Confirmatory Fit Index; RMSEA, Root Mean Square Error of Approximation.

T1, 10 years of age; T2, 12 years of age.

\section{Figures}

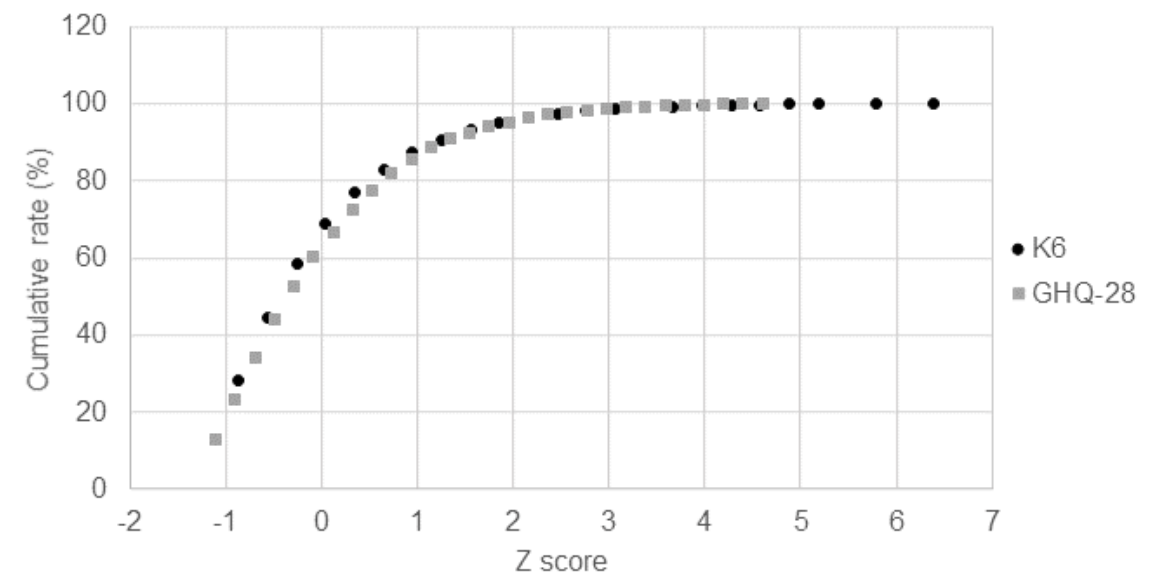

Figure 1

Cumulative distribution of the $\mathrm{Z}$ scores of the $\mathrm{K} 6$ and the GHQ-28 


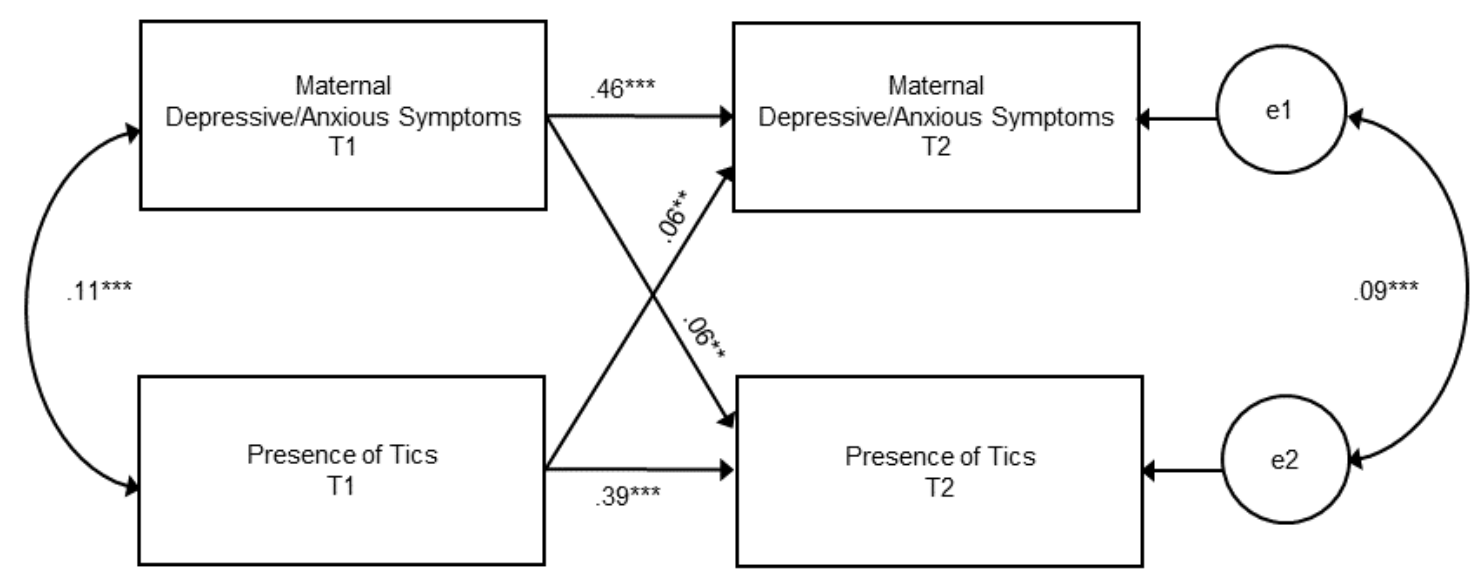

Figure 2

Cross-lagged models of relationships between maternal depressive/anxious symptoms and children's tics. Abbreviations: e, error variable. $T 1=10$ years of age; T2 = 12 years of age. Cross-lagged model of the relationships between maternal depressive/anxious symptoms and children's tics in the populationbased adolescent sample. This figure shows the results of the adjusted model in Table 2. Paths from covariates are omitted from the figure. ${ }^{\star *} p<.01, * \star \star p<$ .001 